ARCHIVO ESPAÑOL DE ARTE, LXXXI, 322 ABRIL-JUNIO 2008, pp. 165-196

ISSN: 0004-0428

\title{
VARIA
}

\section{VAN DYCK: UN NUEVO RETRATO DE AMBROSIO SPÍNOLA IDENTIFICADO EN ESPAÑA}

El Retrato de Ambrosio Spínola de cuerpo entero y armado que restituimos a Van Dyck (fig. 1) ${ }^{1}$, fue estimado original en tesis doctoral, tratando de la Pintura Flamenca del siglo XVII en España en $1976^{2}$, mucho antes de la afortunada adquisición por el coleccionismo madrileño, pero sin las precisiones oportunas, que ahora dispongo, localizándolo y cotejando con las réplicas y versiones conocidas. No fue difícil rectificar la atribución a Juan Carreño de Miranda a cuyo nombre figuraba en 1923, en la Exposición de Arte Antiguo español en el palacio Charpentier de París ${ }^{3}$. Allí constaba el origen en la antigua colección Vendôme y del príncipe Luis Felipe con anterioridad. De hecho, lleva el sello de la colección del Château d'Eu en el reverso del lienzo; pasando al duque de Montpensier en 1902, vendido en $1903^{4}$, atribuido a Gaspar de Crayer en la galería Turquin de París en $1998^{5}$, siendo adquirida aquí.

Una repetición prácticamente exacta, está hoy en la colección Hebsacker de Überlingen (fig. 2) ${ }^{6}$, expuesta en Zurich en 1974 como pieza del taller de Van Dyck. Éste, en la mono-

\footnotetext{
${ }^{1}$ Lienzo, $197 \times 119$ cm., Núm. 141 a la izquierda.

2 M. Díaz Padrón, Pintura Flamenca del siglo XVII en España, Tesis doctoral, Universidad Complutense de Madrid, 1976, Ms. fol. 531, 1122.

${ }^{3}$ Exposition d'Art Ancien espagnol, organisée par la "Demeure Historique”, París, Hotel Charpentier, 1925, p. 11,

4 “A. van Dyck. A Portrait of Count Spinola, Governor General of the Netherlands, in the time of the Spaniards. This portrait once ornamented the Palace Royal at Paris. Engraved. $23 / 4 \mathrm{~h} \times 21 / 2 \mathrm{w}$ ", Charles Farebrother, Londres, 30-IV-1803.

5 Turquin, 24-III-1998.

6 Überlingen, Bodensee, Colección Hebsacker. [M. MenotTI, "Van Dyck a Genova", Archivio storico dell'arte, III, 1897, pp. 442-443; L. Cust, Anthony van Dyck: An Historical Study of His Life and Works, Londres, 1900, p. 243, n. ${ }^{\circ}$ 120; G. GLÜCK, Van Dyck. Des Meisters Gemälde in 571 Abbildungen, Klassiker der Kunst, Stuttgart-Berlin, Nueva York-Londres, 1931, p. 551, n. ${ }^{\circ}$ 295; H. VEY, Die Zeichnungen Anton van Dycks, Monographen des "National Centrum voor de Plastische Kunsten Van XVI de en XVII de Eeuw, Bruselas, 1962.en n. ${ }^{\circ}$ 177; BarNes et alt, Van Dyck, 2004, n. ${ }^{\circ}$ III.A25].
} n. ${ }^{\circ} 9$. 


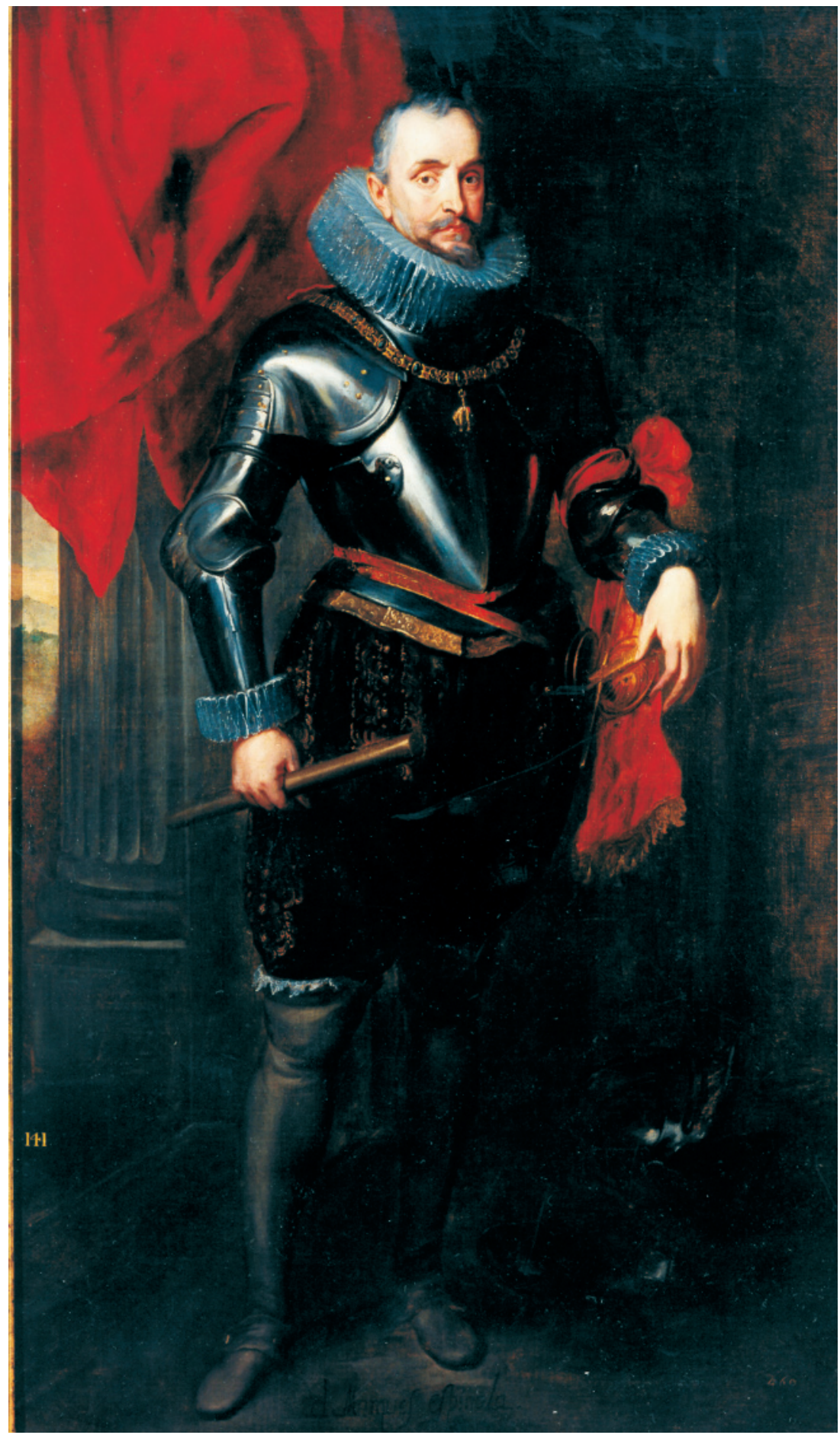

Fig. 1. A. Van Dyck, Ambrosio Spínola, colección privada Madrid.

AEA, LXXXI, 322, ABRIL-JUNIO 2008, 165-196, ISSN: 0004-0428 


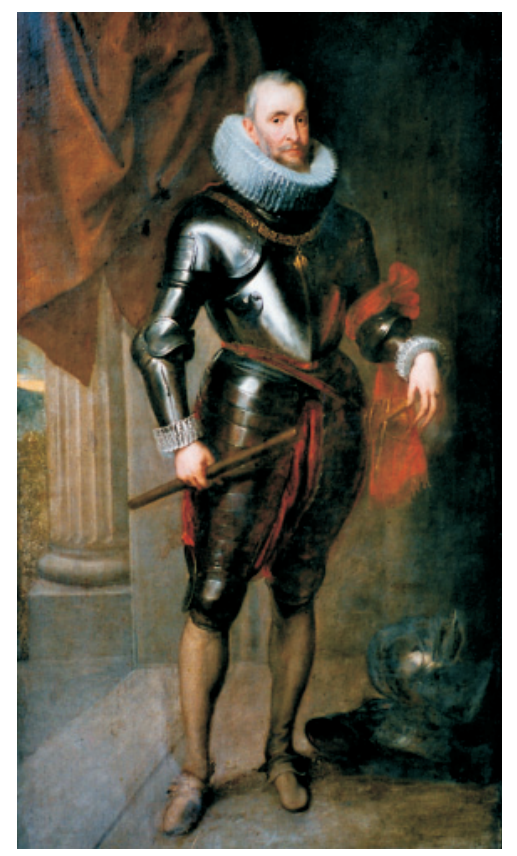

Fig. 2. A. Van Dyck y estudio Ambrosio Spínola, colección Hebsacker \& Hebsacker, Ürbelingen.

grafía última de Van Dyck se cataloga con posibles originales del pintor y taller, réplica de un original que fue enviado a Génova. Se piensa que estuvo en la familia Spínola desde el siglo XVII, pasando a la familia Centurione por herencia, poniéndola en venta en 1903, pasó por sucesivos coleccionistas hasta su propietario actual ${ }^{7}$, a cuya gentileza debo haber podido estudiarla con detenimiento en su residencia de Zurich.

En ambas pinturas el general está retratado de pie con bastón de mando, banda roja ceñida en el brazo izquierdo, y mano apoyada en el pomo de la espada, con gesto y símbolos propios de los capitanes generales del ejército español del siglo XVII. En su pecho pende el Toisón de Oro, distinción que Felipe III le otorgó en 1605. El fondo lo cierra una columna de fuste estriado y un amplio cortinaje con espacio abierto al exterior: es una teatral ornamentación típica de los retratos de aparato de la nobleza del Barroco internacional. El retratado, descansando su cuerpo en el pie derecho y avanzando el izquierdo, domina el primer plano con la mirada fija y arrogante frente al espectador. El foco de luz resbala desde la izquierda sobre el rostro, las manos y la coraza con detalles vibrantes. Es un retrato de poderío y exaltación a las glorias militares que protagonizó Spínola en aquel siglo de dominio español ${ }^{8}$.

7 Principe Centurione Scotto, Génova, Venta Sangiorgi, Roma, 6-V-1903, n. ${ }^{\circ} 44$; Zurich, Dr. Adolf Hommel (Venta Heberle, Colonia-Zurich, 19/20-VIII-1903, n. $\left.{ }^{\circ} 43\right)$, Hippolite Sauer [Zurich, Auktion Nachlass Saber Arbon und Zurich, 14/16-X-1974. Mobiliar ehemals Schloss Eugenberg am Bodensee Solothurn und Zurich, n. 542 (como obra del taller de A. van Dyck)].

${ }^{8}$ Sobre Ambrosio Spínola, véase A. Rodríguez Villa, Ambrosio Spinola, Madrid, ed. 1893, ed. 1904; Idem, Misión secreta del Embajador D. Pedro Ronquillo en Polonia 1674: según sus cartas originales al Marqués de los Balbases, Madrid, [s.a.]; A. CerrolazA, Spinola, un genovés en Flandes; Madrid, 1946; J. Lefevre, Spinola et la Belgique (1601-1627), Bruselas, 1947; H. Herman, Sitio de Breda rendida a las armas de Don Felipe IV... al valor del marqués Ambrosio Spinola, 1998. 
Directa o indirectamente, la imagen toma la pose de la escultura clásica, que Van Dyck utilizó a la par que Rubens ${ }^{9}$. El modelo está tomado desde un punto de vista bajo, análogo a Lomellini con su familia de la National Gallery de Edimburgo y los retratos de medio cuerpo de Federico Enrique de Nassau del Museo del Prado y Palazzo Rosso de Génova y sin bastón de mando, Albert de Ligne de la City Art Gallery de Nueva York.

También Rubens retrató a Spínola de medio cuerpo. Son varios los llegados a nosotros de la Galería de Praga, Herzog Anton Ulrich Museum de Brunswick y Museo de Saint Louis ${ }^{10}$. Van Dyck siguió el esquema de Rubens en lo fundamental, aunque los colores son más sobrios y relajados los gestos, y mayor el efecto del claroscuro con renuncia a ostentosos accesorios de casco y plumas. Van Dyck dota al general de una noble y serena imagen y mayor seguridad ${ }^{11}$.

Nos parece oportuno recordar la amistad y admiración de Rubens por el general. Sus cartas transfieren el retrato psicológico de Spínola, coincidiendo con la imagen de Van Dyck en el retrato que estudiamos: "Es el hombre más sagaz y perspicaz que he conocido. No deja adivinar sus intenciones, no es muy comunicativo, más bien por temor a hablar demasiado que por falta de elocuencia e inteligencia. De su valía yo no digo nada, es suficientemente conocida en todo el mundo. Yo, contrariamente a mi primera impresión, he podido darme cuenta de que es un hombre constante y seguro, y merecedor de toda confianza"12.

Van Dyck captó el talante de este noble general pacifista. Spínola fue el más destacado general español, y responsable de las negociaciones para la Tregua de los Doce Años. Entregado como la archiduquesa y Rubens a conseguir la paz. Fue uno de los generales más brillantes de su tiempo. En reconocimiento a sus servicios, Felipe IV le concedió en 1621 el título de marqués de los Balbases. Luchó por la paz más que por la guerra, en claro enfrentamiento con el Conde-Duque de Olivares. Así, Rubens escribe: “Aquí tenemos algunas prácticas secretas con los holandeses, pero sepa V.E. y créalo cierto, que no hay orden alguna de España para negociar con ellos de alguna forma, a pesar del hecho de que nuestra princesa y el marqués de Spínola están inclinadísimos al bien público (que depende de la paz) como a su propia tranquilidad"13.

España nunca agradeció el esfuerzo personal de este general, cuyos méritos no fueron suficientemente comprendidos en vida, al chocar frontalmente con Olivares en su política belicista.

El estilo del retrato concuerda con el segundo período de Amberes de Van Dyck. Hoy los estudiosos coinciden en fijar la pintura hacia 1627-1628, a la vuelta del pintor de Italia (1627), y antes de la partida del general a España en contacto con Rubens para negociar la Paz (1628).

A juzgar por la antigua fotografía de 1923, y su análisis directo, la réplica de Madrid tenía una franja en lo alto del margen superior, ampliando el formato, con alteraciones en la cortina, y ocultando la vista del paisaje montañoso con una borla añadida. Es comprensible la reserva a la calidad de la pintura en la última monografía del pintor, juzgando por una fotografía antigua de escasa calidad en la Witt Library de Londres e ignorarse una intervención de un restaurador

9 A. BALIS, "Van Dyck's Drawings after the Antique" en Van Dyck 1599-1999. Conjectures and Refutations, Amberes, 2001, pp. 29-42.

${ }^{10}$ The Saint Louis Art Museum [Inv. 33.1934]. A propósito de las diferentes versiones de este retrato véase H. Vlieghe, Rubens Portraits of Identified Sitters painted in Antwerp. Corpus Rubenianum Ludwig Burchard, Londres, 1987, p. 187, n. ${ }^{\circ} 148,149,150$. M. CRAWFORD VOLK, "New light on a seventeenth century Collector, The Marquis of Leganés", The Art Bulletin, 1980, nov. 44, n. ${ }^{\circ}$ 457, p. 261, n. ${ }^{\circ}$ 28, opina que el original es el de Brunswick. El lienzo de Saint Louis es considerado por Vlieghe autógrafo [Vlieghe, op. cit. 1987, n. ${ }^{\circ} 150$ ].

${ }^{11}$ Quizá el precedente de esta fórmula está en los retratos de J. M. Miereveld, como el Retrato de Ambrosio Spínola del Rijksmusem de Ámsterdam.

${ }^{12}$ Carta de Rubens a Dupuy, 23-I-1628 [M. Rooses \& CH. Ruelens, Correspondance de Rubens et Documents épistolaires concernant sa vie et ses oeuvres, 5 vols., Amberes, 1887-1909, IV, p. 358].

${ }^{13}$ Carta de Rubens a Pierre Dupuy, 6-V-1927 [Rooses \& Ruelens, op. cit. 1887-1919, IV, p. 252]. 
desaprensivo que oculto una parte sustancial de pintura del original. Las diferencias del retrato de Madrid con el de la colección Hebsacker no existen sustancialmente después de eliminar los repintes ${ }^{14}$. Las diferencias se reducen a la vestimenta, pues la pintura de Madrid retrata al general medio armado y con calzas, y la de colección suiza lleva armadura completa. También el color de los cortinajes difieren en ambas pinturas: ocre amarillento en la versión suiza, y rojo intenso en la madrileña.

El ancho muro abierto al exterior se desplaza en provecho de la columna y el basamento. El dibujo del fuste de la columna y las estrías son sensiblemente más correctos en el retrato de Madrid. La sombra excesiva de la basa de la repetición suiza y friso con trazos duros, contrasta con el claroscuro más suave del retrato que nos ocupa. También es notoria la ejecución y vigor en los reflejos del yelmo y el suelo. La confrontación de ambos lienzos nos ha permitido reconocer estos matices que legitiman la calidad del retrato de Madrid. Es más perfecto el diseño en la empuñadura de la espada, y está mejor resuelto el codo izquierdo de la armadura, y más preciso el dibujo del Toisón en el pecho. También los toques son más seguros en la gorguera, el rostro más vivo y enérgico en el retrato de Madrid, pero más humanizado el retrato de Überlingen que omite el paisaje del fondo. También el lienzo de la colección suiza acusaba repintes y excrecencias. A pesar de la presumible limpieza continuaba con algunos repintes sin levantar, estucos burdos, saltados de color, y acusados barridos en el yelmo.

A pesar de esto, es espectacular el rojo saturado típico en Van Dyck, los grises metálicos de la armadura, cuya férrea naturaleza metálica captó de manera imponente en los dos retratos.

Van Dyck repitió en varias ocasiones el mismo diseño iconográfico con éxito. Los pliegues de la cortina rescatados debajo de los repintes del retrato de Madrid, viene a coincidir con el de la antigua colección Watney, de Coburn Park de medio cuerpo [fig. 4] ${ }^{15}$, cuyo rostro coincide plenamente con el retrato de pie que estudiamos. En la otra versión de la National Gallery de Edimburgo, que se tiene por taller, el general lleva armadura completa, junto a una mesa sobre la que está el casco [fig. 5] ${ }^{16}$. El busto oval en el Sterling and Francis Clark Art Institute de Williamstown de Massachusetts [fig. 6] ${ }^{17}$, acusa reflejos más vibrantes en la armadura, similares

${ }^{14}$ En la restauración que tuvo lugar en 1998 se desmontó el lienzo del bastidor, se eliminaron las bandas añadidas con posterioridad a la ejecución (pasando de $213 \times 120 \mathrm{~cm}$. a sus medidas actuales), se sentó el color en las zonas perdidas, se limpió, se eliminaron antiguos repintes, estucando pequeñas lagunas.

15 Paradero actual desconocido. Procede de O. V. Watney, Cornbury Park, Inglaterra (Catalogue of the Cornbury Collection, 1915, n. ${ }^{\circ} 18$ ); Christie's, Londres, 23-VI-1967, n. 22 (como Van Dyck); Expuesto en 1968 [London, Agnew's, Sir Anthony van Dyck, A Loan Exhibition of Pictures and Sketches Principally from Private Collections in Aid of the National Trust Neptune Appeal and the King's Lynn Festival Fund, 1968, n. ${ }^{\circ} 24$ (como Van Dyck); Col. Edward Speelman Ltd (1968-1988); Pasó en venta Christie's, 26-I-2001, n. ${ }^{\circ} 123$ (como Taller de Van Dyck); Christie's, 11-VII2001, n. 17 (como Sir Anthony van Dyck y taller). Fue considerado como original de Van Dyck por Larsen, L'Opera completa di Van Dyck, ed. Rizzoli, Milán, II, 1980, n. ${ }^{\circ}$ 430; Idem, The Paintings of Anthony van Dyck, 2 vol., Freren 1988, II, p. 167, n. ${ }^{\circ}$ 40], por J. MüLLER HofsTEDE, "Neue Beiträge zum oeuvre Anton van Dycks", Wallraf-RichartzJahrbuch, XLVIII-XLIX, 1987-88, pp. 175-177]; y por M. JAFFÉ (según señala el catálogo de la galería Christie's). Vey, en cambio, lo consideró repetición o copia de un original perdido de cuerpo entero (en carta del 9-IX-1999). En la última monografía del pintor se considera repetición parcial del lienzo de Überlingen [BARNES et alt., Van Dyck 2004, p. 410]. Pensamos que es lienzo de buena calidad. Los arrepentimientos en el brazo derecho y en el bastón de mando apuntan a esta consideración, a pesar de ciertas debilidades en el atuendo.

${ }^{16}$ Edimburgo, National Gallery of Scotland. Tradicionalmente atribuido a Sustermans. Larsen lo considera obra de taller con reservas [LARSEN, op. cit. 1980, I, p. 116, n. ${ }^{\circ} 431$; LARSEN, op. cit. 1988, II, p. 433, n. ${ }^{\circ}$ A71], y señala su procedencia del Palazzo Gentile de Génova (1830) (mencionada por Menotti 1897, p. 442; GLÜCK, op. cit. 1931, nota en p. 551, p. 295; Catalogue Edimburg 1957, n. ${ }^{\circ} 87$; VEY, op. cit. 1962, n. ${ }^{\circ}$ 177, ill. VIII; 1648. War and Peace in Europe, Cat. Exp. Münster/Osnabrück, 1998-1999, p. 349, n. ${ }^{\circ}$ 995; BARNES et alt, Van Dyck 2004, p. 412 (como variante de taller).

17 Procede de la colección de Lord Radstock; pasando a W. H. WaYne; y R. Kann, París. [W. Bode, Die Gemäldesammlung des Herrn Rudolf Kann, Viena, 1900, pl. 69, XXIV; Rooses, Vijftig meesterwerken van Antoon van Dyck, Anvers, 1900, pp. 26-28; GLÜCK, op. cit. 1931, p. 295; LARSEN, op. cit. 1980, p. 116, n. ${ }^{\circ}$ 429; LARSEN, op. cit. 1988, II, 


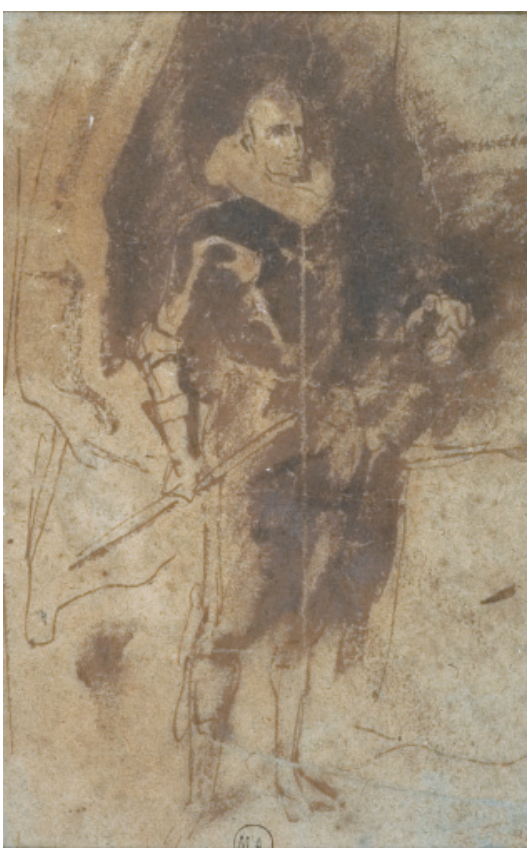

Fig. 3. Museo Atger, Montpellier. Dibujo.

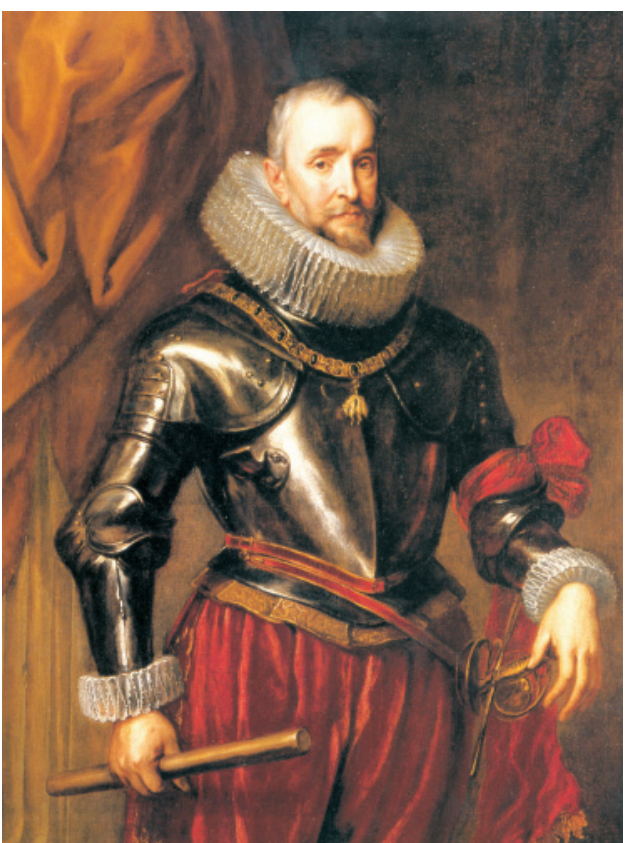

Fig. 4. A. Van Dyck Ambrosio Spínola, Antigua colección O. V. Watney.

a la versión madrileña y distantes del brillo de factura más lamida en la armadura de la colección Hebsacker.

El dibujo preparatorio del Museo Atger de Montpellier [fig. 3$]^{18}$ es para nosotros el documento más alentador para la identificación del retrato de colección madrileña. Vimos que corresponde con mayor exactitud a esta réplica, pues el general lleva calzas. Debió haber previsto la mesilla del lado izquierdo, y marco en el fondo, a los que renuncia en la versión final en pintura (aunque sí en la de Edimburgo), así como la amplitud mayor del cortinaje. Redujo el tamaño del bastón de mando, que es más largo en el dibujo, y corrigió la posición del pie izquierdo, que colocó en disposición más vertical en el lienzo definitivo. Es un excelente dibujo logrado a base de toques precisos con efectos lumínicos: un alarde de virtuosismo y maestría.

Existe grisalla de Lucas Vorsterman en Boughton [fig. 7. . $^{19}$ para el grabado de Vorsterman que difundió la imagen del general [fig. 8 $]^{20}$. Otro diseñó Peter Soutman y esculpió Jacob Louys

p. 167, n. ${ }^{\circ}$ 408; List of Paintings in the... Clark Art Institute, Williamstown, 1992, p. 42; BARNES et alt., op. cit. 2004, p. 412, donde se menciona una copia en Perth].

${ }^{18}$ Lleva la firma: Wandyck fecit. Montpellier, Musée Atger, n. ${ }^{\circ}$ 141. Musée Atger, Montpellier, inv.nr. MA 474 [Citado por CH. SAunier, "Le Musée Xavier Atger à Montpellier", Gazette des Beaux Arts, 1922, I, 172; GlücK, op. cit., 1931, p. 295; Vey, op. cit. 1962, p. 247, n. ${ }^{\circ}$ 177; C. en P. NICQ, Petits et Grands Maîtres du Musée Atger - II. Dessins italiens et nordiques des XVIe. XVIIe et XVIIIe siècles, Montpellier 1997, nr. 87, il. En color, como retrato de anciano].

19 Boughton House, colección del duque de Buccleuch. Expuesta en Works of Art from Midland Housees, Birmingham, 1953, n. ${ }^{\circ} 119$; Vlamse Kunst, Londrews, Royal Academy, 1953/54, n. ${ }^{\circ} 492 \mathrm{e}$.

${ }^{20}$ Con la siguiente inscripción latina: "ILLVSTRISS.[MVS] PRINCEPS AMBROSIVS. SPINOLA. MARCHIO. SESTI. / ET. VENAFRI. DVX. SANSEVERIN. EQ. AVR. VELLER. ARMOR. ET. EXERCIT. / CATH. [AE]. MAI. [TIS] IN. BELG. PRAEFECT. ET. GVBERNAT. GNALIS". L Vorsterman culp (abajo a la izquierda); Ant. Van Dyck pinxit (en el centro); Mart. vanden Enden excudit Cum privilegio. Existe una copia del grabado por un autor anónimo. 


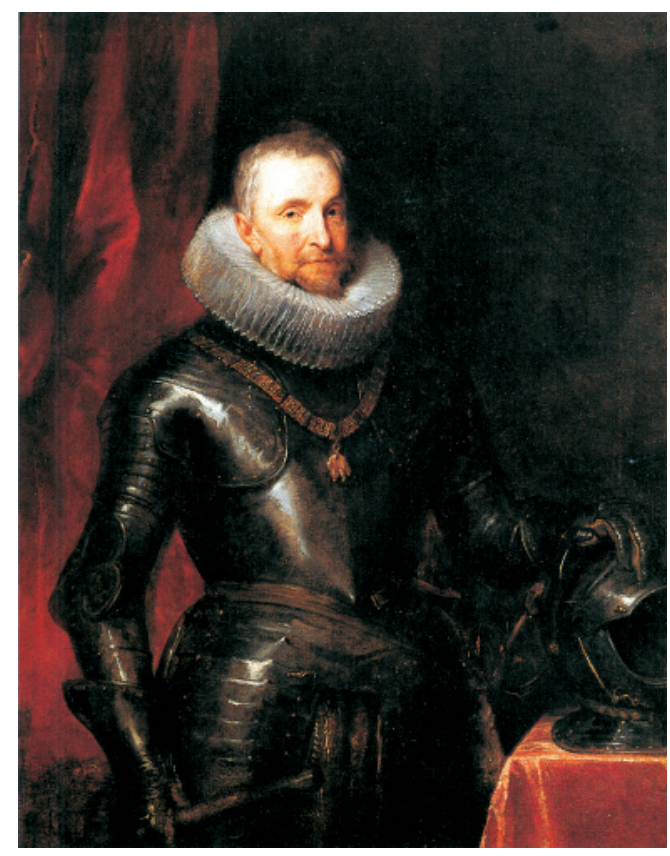

Fig. 5. A. Van Dyck Ambrosio Spínola, National Gallery of Scotland, Edimburgo.

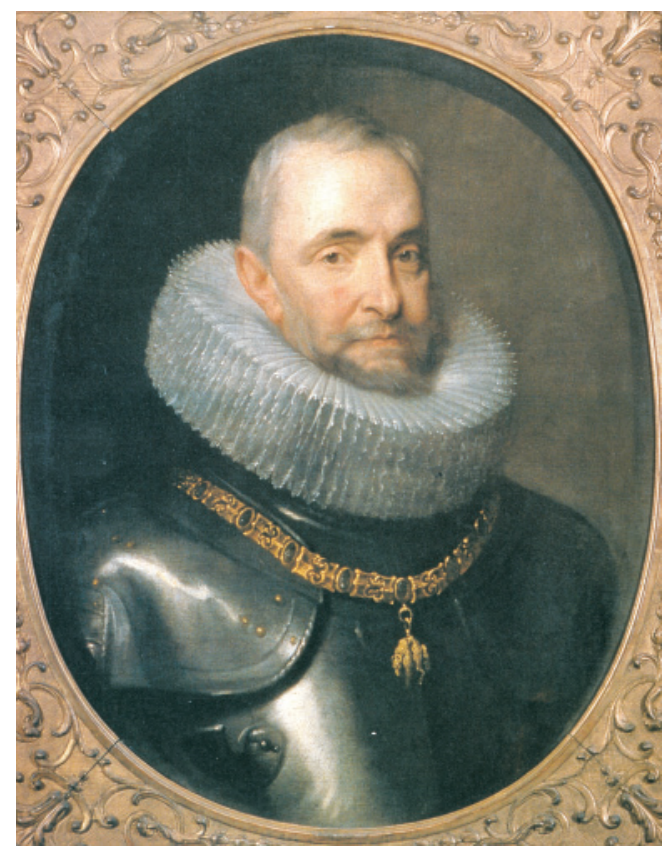

Fig. 6. Taller de Van Dyck, Ambrosio Spínola, Sterling and Francis Clark Art Institute, Williamstown, Massachusetts.

[fig. 10] $]^{21}$ con armadura y rodeado de una guirnalda con motivos vegetales y frutas, y cartela inscrita en la parte inferior. La composición coincide fundamentalmente con un dibujo también atribuido a Soutman en el comercio [fig. 9 $]^{22}$. De una copia del rostro, en papel pegado a tabla, conocemos una antigua fotografía ${ }^{23}$.

Cabe preguntarse sobre la procedencia más remota del retrato que apuntamos en posesión de Luís Felipe. Las colecciones españolas del siglo XVII, registran varios retratos del general, pero no hay pruebas sólidas que puedan corresponderse con el que nos ocupa. Un retrato poseyó el marqués de Leganés, su yerno, según su inventario post-mortem de $1655^{24}$. No dan las medidas en el inventario pero, a juzgar por la tasación, debía tratarse de un retrato de medio cuerpo. Pasó en el siglo XIX a sus herederos, los duques de Altamira. También el duque de Aremberg, que reunió en

[New Hollstein-Van Dyck, I, n. ${ }^{\circ}$ 30]. Menciona una grisalla, y un dibujo de Vorsterman, fechado en 1620, en Londres (Hind, II, 146, N. ${ }^{\circ}$ 5, taf. 76; Vey, op. cit. 1962, p. 248).

${ }^{21}$ En la cartela se lee la siguiente inscripción, en cuatro líneas: “Ambrosius Spinola Princeps Illustrisimus et Excellentisimus / Marquis festi et Venafri, Dux Sanseverinus / Eques Aurei Velleris, Regi Catholico a Consiliis status / Et Belli Militiae, et Aerary Regy In Belgio Prefectus". Abajo a la izquierda: “Ant. Van Dÿck/ Pinxit. Centro: Cum Priuil. Sa. Ca. M. / P. Soutman Effigiauit et Excud. Derecha: I. Loü̈s / Sculpsit [Vid. New Hollstein-Van Dyck, part IV, p. 107, n. ${ }^{\circ} 270$, p. 120].

22 París, Christie's, 15-XII-2004, n. 254 (atribuido a Pieter Claesz. Soutman).

${ }^{23}$ Estuvo en colección privada suiza, y en la colección Holmquist de Estocolmo (1938); pasó en venta Triberg, Nueva York (Colección Kende, 18-V-1950, n. $\left.{ }^{\circ} 20\right)$.

24 N. ${ }^{\circ}$ 449: "Ambrosio de Espinola, marques de los balbases 607v armado, de mano de Vandick, del mismo tamaño la taso en 200”. 1655. Testamentaría del marqués de Leganés [Vid. Poleró 1898, n. ${ }^{\circ}$ 64, p. 132; López Navío 1962, n. $\left.{ }^{\circ} 449\right]$. 


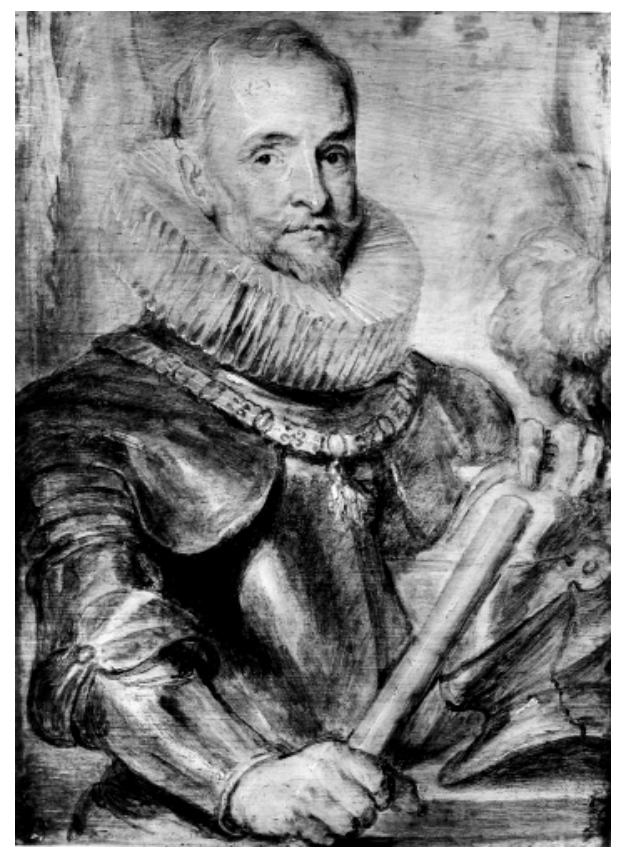

Fig. 7. Lucas Vorsterman según Van Dyck, Grisalla, Boughton House, colección del Duque de Buccleuch.

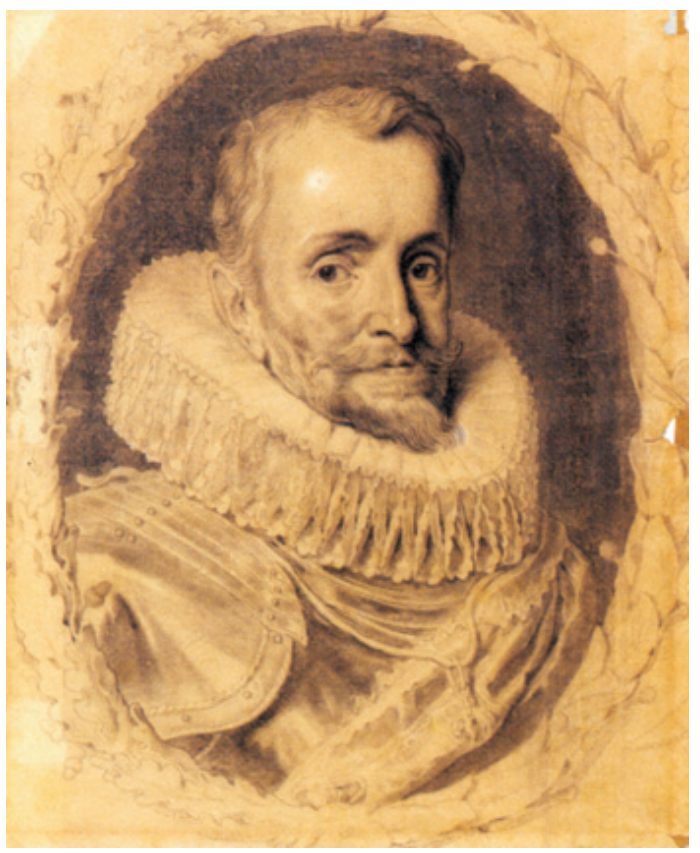

Fig. 9. Atribuido a Peter Claesz. Soutman. Dibujo.

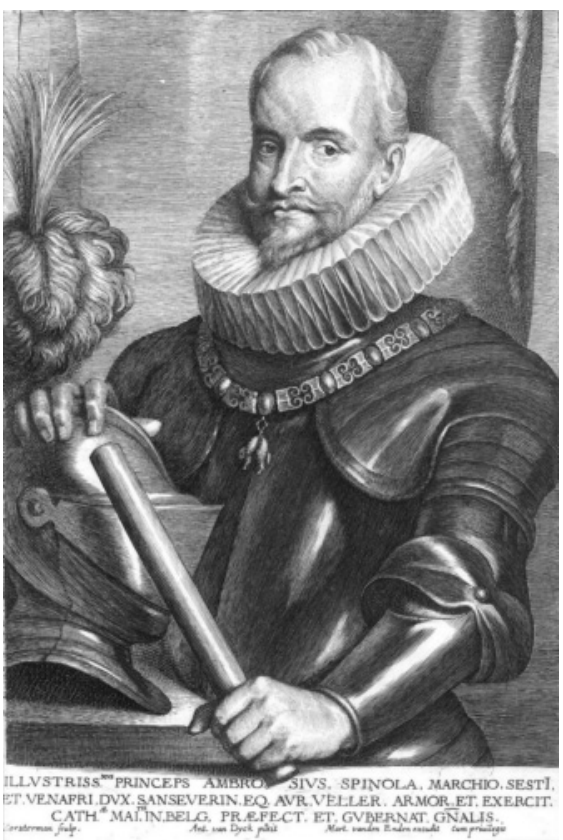

Fig. 8. Lucas Vorsterman según Van Dyck. Grabado.

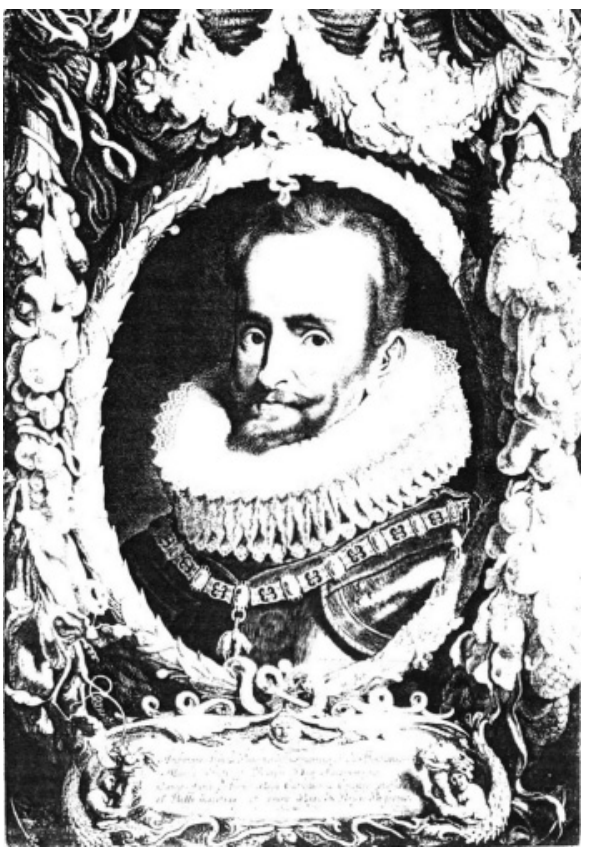

Fig. 10. J. Louys según Van Dyck. Grabado. 
su casa de Madrid una espléndida galería de retratos de personajes ilustres, cita dos de Ambrosio Spínola, uno a caballo y otro en pie, en inventario de 1641 , pero sin mencionar los autores ${ }^{25}$. A nombre de Van Dyck constan otros dos en colecciones privadas de Ámsterdam en el siglo XVII ${ }^{26}$, y en Amberes, en la colección del orfebre Jan Gillis ${ }^{27}$.

También son muy numerosos los retratos de Ambrosio Spínola en colecciones italianas, pero muchos de ellos nos llegan sin autor. A nombre de Van Dyck, uno de medio cuerpo, con armadura y bastón de mando poseyó Francesco Maria Balbi en Génova, según su testamentaría de $1701^{28}$, y ventas hasta principios del siglo XIX, sin el que nos ocupa pueda identificarse con seguridad. Otros del mismo personaje, sin mayor precisión, localizamos en diversas ventas ${ }^{29}$.

\author{
Matías DíAz PADRón \\ Museo del Prado
}

\begin{abstract}
25 "Seis Liencos de Tres varas de alto y dos varas y media de ancho q Son El Marques de espinola a Cavallo = El Conde bucoya a Cavallo El Marques de leganes a cavallo = El conde de fuentes a Cavallo: El Conde de arenverg Visabuelo de su Ex ${ }^{\mathrm{a}}$ y El Conde de tilli Con Sus marcos Tasado Cada uno en Ciento y Treinta ducados que Todos montan nueve mil y novecientos rreales 9900. [AHPM, Prot. 5.993, ff. 1224-1238v, Burke \& Cherry 1997, pp. 345-358, 77. ${ }^{a}$ ]. "Treinta y nueve rretratos en pie de a dos varas Y media de alto y vara y Tercia de ancho que son Los que se siguen El Rey de Yngalaterra y su mug ${ }^{\mathrm{r}}-\mathrm{El}$ archiduq ${ }^{\mathrm{e}}$ hernesto $=$ El enperador Padre del moderno y Su muger $=$ El rrey de francia $\mathrm{Y}$ Su muger $=$ Don carlos y El ynfante Cardenal $=$ Una pariente de su $\mathrm{ex}^{\mathrm{a}}-\mathrm{El} \mathrm{duq}^{\mathrm{e}}$ de telli $=$ Una hermana del Enperador de oy. El Rey de polonia con su mugr - rrodolpho Enperador $=$ El archiduque Alberto y Su mug ${ }^{\mathrm{r}}=$ El rretrato de maris-

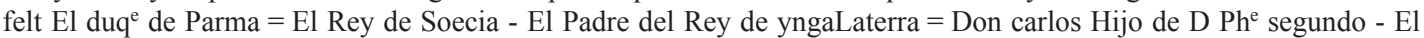
Conde de fuentes $=$ Hermano del archiduq $^{\mathrm{e}}$ Alverto $=$ mathias enperador hermano del mismo $=$ El duq de orlens y su dos mugeres $=$ otra parienta de su $\mathrm{Ex}^{\mathrm{a}}=\mathrm{El}$ rrey de françia Padre del de oy y Su muger $=$ frislan $=\mathrm{El}$ Conde de olivares

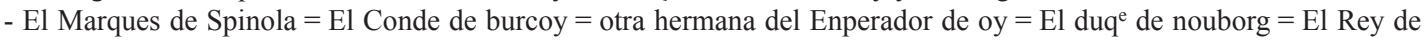
Polonnia de oy Tasados Cada uno en Sesenta Ducados 25740" [f. 1230-1230v 37; Ibidem, n. ${ }^{\circ} 78 \mathrm{zi}$ ].

26 "'t Contrefeijtsel van Spinola"; Inventario de Marry Jacobs, viuda de Jan Claesz. Hollesloot, 30-VIII-1630 - 3IX-1630, [Inventaris ende specificatie van allen den goederen nagelaten by z. Marry Jacobs weduwe ende boedelhouster was van wylen Jan Claesz, Gemeentearchief, Amsterdam, Nederland (NAA 568, filmnrs. 4919 \& 6547, ff. 303-321, Getty Archival Document N-2305]. Seguramente se trate del mismo que se registra en venta medio siglo después: "Een contrafijtsel van de marquis Sijnola in vergulde lijst van den heer Van Dijck 120"; Rinaldi, Louis (verkocht aan Jacques la Vesne), 10 February 1684, Amsterdam, Netherlands, Gemeentearchief, Amsterdam, Nederland (NAA 3472 (film 3508) ff. 53-54), Getty Description of Archival Document N-1261.

27 E. Duverger, Antwerpse kunstinventarissen uit de zeventiende eeuw, Bronnen voor de Kunstgeschiedenis van de Nederlanden, Bruselas, XI vols, XI, doc. 28/30-VII-1682.

28 “36 Un Quadro d'un ritratto d'un Signor Spinola mezza figura vestito d'armi dure con bastone di comando del Vandich”, 20-XII-1701: Testamento en beneficio de Francesco Maria y Constantino Balbi. [Nota de quadri che sono al presente in Casa del Signor Francesco Maria Balbi Seniore ripartiti in ogni stanza, Archivio di Stato, Genova, Italia (f. 5, anni 1700-1702, 20 dicembre 1701] P. Boccardo \& L. Magnan, "La famiglia dei Balbi: la committenza" Il Palazzo dell'Università di Genova. Il Collegio dei Gesuiti nella strada dei Balbi, Università degli Studi di Genova, 1987, pp. 47-88. Vendido en Londres en 1807: “A. van Dyck. The Portrait of General Spinola. This portrait is arrayed partly in armour, and the right-hand holds a marshal's truncheon, emblematical of command, while the countenance of the warrior is depicted with appropriate character, and in the happiest stile of Vandyck's art. It displays with due energy the man of understanding, and the cool and collected, yet determined soldier, at once animated and dignified. This Picture was considered the chef d'oeuvre of Van Dyck in Genoa. From the Balbi Palace, 4 1/2 $h \times 4$ w", Peter Coxe, Londres, 6-V-1807. Vendido de nuevo en Londres en 1823: "Vandyke. Portrait of Spinola, from the Balbi Palace", Phillips, 19-IV-1823. Se vende de nuevo en 1826: "V. Dyck. Portrait of the celebrated Genoese General, Ambrogio Spinola, in plate armour, grasping his truncheon with one hand, and with the other supporting the hilt of his sword, in a commanding and energetic attitude, and the head painted with great spirit and elegance: a noble specimen of this unrivalled Painter of Portrait. From the Balbi Palace at Genoa - 4 ft. 6 inches high, by 3 ft. 11 1/2 wide”, Christie's, 12-V-1826 (comprado por Alexander Baring).

29 "Portrait of General Spinola", Christie's, Pall Mall, Londres, 21-I-1815, n. ${ }^{\text {o } 36 . ~ " P o r t r a i t ~ o f ~ S p i n o l a " ~(v e n d i d o ~}$ por Michael Brian), Christie's, 16-VI-1820. "Portrait of the Marquess Spinola of Genoa”, Christie's, 20-II-1830.
\end{abstract}

